\title{
Categorical Perception of Colour is Lateralised to the Left Hemisphere: Evidence from Present-Absent and Pop-out Tasks
}

\author{
Abdulrahman Saud Al-Rasheed
}

\begin{abstract}
Left Hemisphere LH bias in colour Categorical Perception $\mathrm{CP}$ has been related to the linguistic nature of the LH and converging evidence to support this hypothesis has been presented. So far all studies have used either the visual search task or the target detection task with most of these tasks involving a spatial decision about whether the target is on the left or the right. Current study extended the investigation to include two other types of tasks. First a search task that varied the number of distractors and second a visual search task with targets only present on half the trials and the task was to decide whether there was a target present or not. Forty native-English-speaking participated in this study. The pattern of $L H$ colour $C P$ was found on both of these tasks. This result has been established that $\mathrm{LH}$ colour $\mathrm{CP}$ is a solid phenomenon
\end{abstract}

Index Terms-Left hemisphere, lateralization, categorical perception, visual search task, target detection task.

\section{INTRODUCTION}

The colour spectrum is a physical continuum but it is perceived discontinuously, as discrete categories or segments of hues [1]. This is part of an effect called Categorical Perception (henceforth, CP). CP is found when a continuum is divided into categories, and when these categories appear to affect discrimination. In operational terms, $\mathrm{CP}$ can be defined by faster and/or more accurate discrimination of pair of stimuli that cross a category boundary (across-category), than two stimuli from the same category (within-category), even when the stimulus differences between the pairs of stimuli are equal.

Evidence for $\mathrm{CP}$ has been reported on a wide range of colour perception tasks. For example, recognition memory and X-AB tasks, [2]-[6] same-different tasks [7], [8] similarity judgments [3], [9] and target detection and visual search tasks [10]-[13] To investigate the contribution of language to $\mathrm{CP}$, recent studies have considered how the effect is lateralized, [14]-[17]. Gilbert et al. reasoned that, as the left hemisphere is dominant for most language functions, if colour $\mathrm{CP}$ is related to language it should be stronger in the LH. To test this, Gilbert and colleagues used a visual search task where targets were lateralised to the left or right visual field (LVF/RVF). Stimuli were shown in a display of twelve coloured squares in a clock shape; eleven of the squares (the distractors) were identical in colour, and one (the target) was different. The relationship between the distractors and the

Manuscript received June 9, 2014; revised October 18, 2014

Abdulrahman Saud Al-Rasheed is with the Department of Psychology, King Saud University, Riyadh, KSA (e-mail: asalrasheed@ksu.edu.sa). target stimulus was manipulated so targets and distractors were either from the same colour category (e.g., blue1-blue2 or green1-green2), or from a different colour category (e.g., blue1-green1 or green1-blue1). While looking at a central fixation cross, participants had to decide whether the target was to the left or to the right of fixation. Gilbert et al. found that RTs were faster when target and distractors were different categorically (blue1 among green1s) than when target and distractors were just perceptually different (blue1 among blue2s). However, this category effect was found only if the target was presented to the RVF. Gilbert et al. argued that this pattern of lateralisation was consistent with $\mathrm{CP}$ being due to the implicit use of language.

The first replication of Gilbert et al. [14] came from a re-analysis of a previous visual search study conducted by Daoutis, Pilling and Davies [12]. They used a visual search task that required the detection of a target colour amongst two kinds of distractors. A target was only present on half of the trials and the task was to decide as quickly as possible if the target was present. Although on target present trials, half the time the target appeared in the LVF and half the time in the $\mathrm{RVF}$, in the original paper, the possibility of visual field effects had not been considered. A reanalysis including visual field as a factor showed a stronger categorical effect for targets appearing in the RVF than for those appearing in the LVF. Drivonikou and colleagues [18] then investigated whether lateralised $\mathrm{CP}$ would be found in a simplified version of Gilbert et al.'s search task, where there was a single target colour on a background of a different colour (see Franklin et al. [19]). Participants had to detect a circular coloured target that appeared in one of 12 locations on a coloured background. The target and background were from either just perceptually different (e.g., blue1 among blue2s) or physically and categorically different (e.g., blue1 among green1s) with the target-background perceptual distances equated across conditions. The results showed that RTs were faster when target and background were categorically different, than when they were just perceptually different This category effect was found in both visual fields, but was larger in the RVF than LVF. In the same study, Drivonikou et $a l$. also tested the blue-purple category boundary, and again, a category effect was found in the RVF, but not this time, in the LVF.

There is also evidence that LH lateralised colour CP only occurs if the category boundary is marked in the language. Korean has a lexical boundary between yeondu (yellow-green) and chorok (green) that is not marked in English. Roberson et al. [16] compared English and Korean speakers using Gilbert et al.'s [14] visual search task where 
the target-distractor relationship was either within-yeondu or -chorok, or between yeondu and chorok. CP was shown by Korean participants but not English participants, but there was no visual field by category interaction (the usual signature of lateralised CP). However, dividing the Korean group into fast and slow responders, using a median split, revealed that $\mathrm{CP}$ was lateralised to the RVF-LH for fast responders, but was present in both VFs for slow responders. Roberson et al. suggested that for slow responders, there was sufficient time for information to be transferred from the $\mathrm{LH}$ to the RH across the corpus callosum allowing language to influence performance in both visual fields.

Another cross-cultural study by Drivonikou, Davies, Franklin and Taylor [18] compared hemispheric asymmetry in colour CP for three samples: Greeks, English and 'Africans'. The same target detection task as in Drivonikou et al. [16] was used. Greek and English were tested for a category effect across two Greek basic colour categories ble 'dark blue' and galazjo 'light blue' which is not marked in English. A category effect was found for Greeks but not for English; moreover, for Greeks, the category effect was lateralised to the LH. The same task was used to test English and African participants for a category effect across the English blue-green boundary that is not marked in the various languages spoken by the African group. A category effect was found for the English group but not for the African group and for the English group, it was stronger in the LH than the RH.

Lateralisation of colour $\mathrm{CP}$ to the $\mathrm{LH}$ has been also been investigated using functional magnetic resonance imaging fMRI [20] and the event-related potential (ERP) technique [21]. In Siok et al. Chinese participants' brain activity was scanned while they performed a visual search task. The task, procedure and design were the same as Gilbert et al. 's. [14]. There was stronger activity in the language regions of the brain (the posterior temporoparietal area, the middle temporal gyrus and the inferior prefrontal cortex) in the left cerebral hemisphere for across- than for within-category discriminations in the RVF. This was also associated with greater activation in visual cortex for across- than for within-category discriminations. Liu et al. [21] tested 12 adult Chinese on the same visual search task. N2pc (N2-posterior-contralateral) was used as an index of the attentionional demands of within- and across-category target-distractor relationships in the visual search task. The $\mathrm{N} 2 \mathrm{pc}$ components in the LH were larger for the cross-category condition than for the within category conditions.

So far, the LH bias in colour CP has been related to the linguistic nature of the $\mathrm{LH}$ and converging evidence to support this hypothesis has been presented. However, as, to date, no studies of lateralised CP have been conducted using other than either the visual search task of Gilbert et al. or the target detection task, with most of these tasks involving a spatial decision about whether the target is on the left or the right.

\section{Aim of the Study}

The aim of this paper is to establish whether the LH colour category effect is unaffected by seemingly small scale procedural variations. So far, all studies have used either the visual search task of Gilbert et al. or the target detection task, with most of these tasks involving a spatial decision about whether the target is on the left or the right. Experiment 1 and 2 extended the investigation to include two other types of tasks. First, a search task that varied the number of distractors. And second, a visual search task with targets only present on half the trials, and the task was to decide whether there was a target present or not, rather than decide the location of the target. The aim here was to see whether this colour search task exhibited 'pop-out' [22] or 'efficient search' [23].

\section{EXPERIMENT 1: HEMISPHERIC ASYMMETRIES IN COLOUR CP AND 'POPOUT': THE EFFECT OF NUMBER OF DISTRACTORS}

A previous study by Al-rasheed et al. [17] investigated whether the $\mathrm{LH}$ bias in colour $\mathrm{CP}$ was independent of reading direction. The results showed that lateralised $\mathrm{CP}$ was independent of habitual reading direction and broadly replicated previous results, [14], [15], [24]. Most tests of lateralised CP have used one or other of these tasks, and it is important to establish that the effect is independent of the detailed methods used. Here, we investigate first varying the number of distractors affects lateralised CP (Experiment 1) and then whether removing the spatial decision (left or right of fixation) affects lateralised CP (Experiment 2).

Most of the studies that tested the lateralised CP effect used tasks similar in design to the original study by Gilbert $e t$ al. [14]. In the next experiment (Experiment 1), the number of distractors was varied to test whether search time was independent of the number of distractors, indicating 'pop-out' [22], or that search was 'efficient' [23]. If search was not efficient, then the pattern of lateralisation might interact with the number of distractors. In Experiment 2, targets were only present in half the trials, and the task was to decide whether there was a target or not. Thus, this Experiment tested whether the same pattern of lateralisation was found as in Gilbert et al. [14] when the explicit spatial component of the decision was removed. As the results from Al-rasheed [17] showed that the pattern of lateralisation was independent of reading direction, the following experiments only tested an English-speaking sample. Al-rasheed [13] establish the location of the Arabic azrock 'blue'- akhdar, 'green' category boundary and confirmed that the boundary was at about 7.5BG as for English [25].

\section{A. Method}

\section{1) Participants}

Sixteen native-English-speaking undergraduates from the University of Surrey participated in this experiment. There were 4 males, with a mean age of 24 years $(\mathrm{SD}=8)$, and 12 females, with a mean age of 20 years $(\mathrm{SD}=6)$. Their ages ranged from 18 to 24 years. Based on self-report, all were right-handed and had normal colour vision as indicated by the City University Test [26]. Most of the participants participated for course credit and a few volunteered.

\section{2) Stimuli and apparatus}

As shown in Fig. 1, three colour stimuli were used in this experiment; one green (5BG) and two blues (10BG and 5B; 
Value and Chroma $=6 / 8)$. The separation between adjacent stimuli was 5 Munsell hue steps (AE 15). Their CIELUV coordinates $\left(u^{*} v^{*}\right)$ were $-45.65,-2.73 ;-44.83,-18.68 ;-39.62$ -32.97; all at $L^{*}=61.70$; a Cambridge Research Systems, ColourCal colourimeter was used to measure the CIE co-coordinates and they were displayed on a 17-inch CRT model GDM-F520.

\section{3) Procedure}

Adjacent stimuli were paired, to form one within-category pair (blue1-blue2) and one between-category pair (blue1-green1). For each pair, one stimulus was the target and the other stimulus was used for the distractors, with both stimuli in a pair appearing equally often as distractors. The target for all trials was always blue and the distractors were randomly switched between 'within' (blue) and 'across' (green). There were equal numbers of trials for each combination of category (within- or between-) and visual field (LVF or RVF) and the order of trials was randomised across these four conditions. In addition, target location was randomised across trials with the constraint that the target appeared equally often to the left and right of fixation. Stimuli were shown as $2.5 \mathrm{~cm}$ squares with $5 \mathrm{~mm}$ gaps between adjacent locations, appearing in locations specified by a $6 \times 6$ square grid on the display (Fig. 1). The target appeared amongst either 3,15 or 35 distractors on a grey background $\left(19.47 \mathrm{~cd} / \mathrm{m}^{2}, 0.336,0.344\right)$. For the 4 and 16 set sizes (distractors plus target) stimulus locations within the grid were randomly selected, but for the 36 set size, all locations were occupied (see Fig. 2).

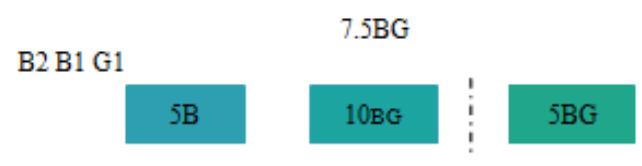

Within-category Cross-category

Fig. 1. Illustration of the Munsell codes of the three stimuli used. Stimuli B1 and B2 are from the same category, while stimulus G1 belongs to a different category. B1-B2 and B1-G1 differ by 5 Munsell Hue steps. The line between B1 and G1 indicates the English and Arabic blue-green boundary.

The experiment began with a fixation cross which remained for $100 \mathrm{~ms}$ to alert the participants that the trial was beginning. Then the test display followed and remained on screen for $200 \mathrm{~ms}$. The next trial began when the participants had responded. There were 192 trials, 16 for each combination of category and visual field, repeated three times, once for each set size. Participants were given 10 practice trials before starting the experiment, and they took about ten minutes to complete the task.

The participants were tested individually in a dark room and sat with their head position constrained by a chin-rest, so that eye-level was at the centre of the monitor, with a viewing distance of $60 \mathrm{~cm}$. Participants were informed that they would be presented with a target stimulus among a varied number of distractors and their task was to decide whether the target was to the right or to the left of fixation. Responses were made by clicking the appropriate mouse button.

\section{B. Results}

The percentage of incorrect trials was calculated for each subject, for each combination of category (within/cross) and visual field (left/right) and number of distractors (3, 15 or 35). A three-way repeated measures ANOVA on the error rates showed that there were no significant effects. However, the number of distractors was almost significant, $F(2,28)=3.13$, $p=0.059$. (means $(3 \mathrm{D})=1.133,(15 \mathrm{D})=0.733,(35)=$ $0.917)$.

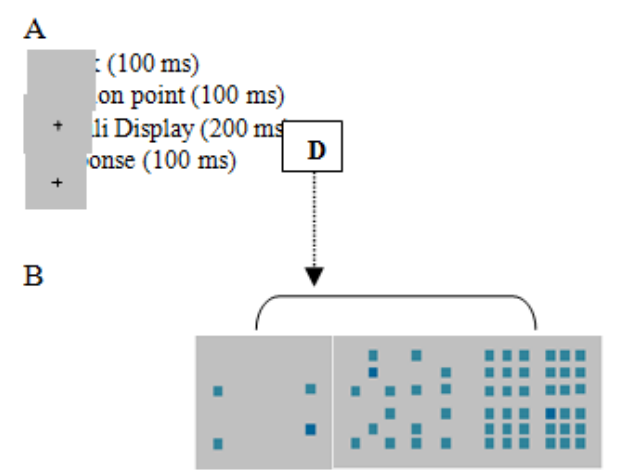

Fig. 2. a) Example of the grid task. The blue square shows the target, and the other green squares indicate the distractors. b) Illustration of the three set of distractors $(3,15$ and 35$)$ for the 3 and 15 stimulus locations within the grid were randomly selected, but for the 36 set, all locations were occupied.

Then, for each participant, median RTs for correct trials for each combination of category visual field and number of distractors were calculated. A three-way repeated measures ANOVA showed that there was no effect of the number of distractors nor did it interact with any other factor (maximum $F=1.01$ ). Fig. 3 shows the mean RTs for each combination of category and visual field collapsed across the number of distractors.

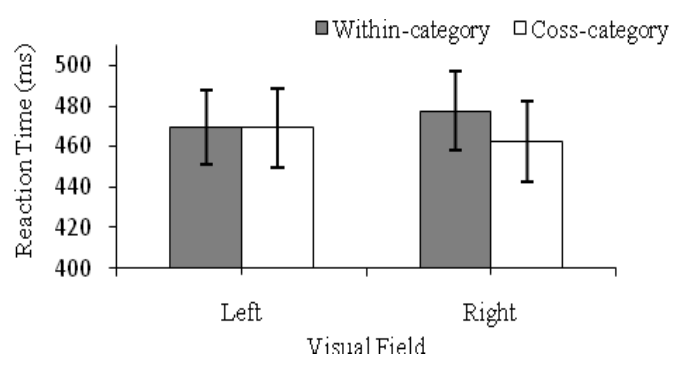

Fig. 3. Mean response times (+/-1se) for each combination of category and visual field.

Although between category RTs were only about $6 \mathrm{~ms}$ faster than within-category RTs, this difference was significant (Means $(\mathrm{SD})=468.98(73.75) \mathrm{ms}, 474.89$ (77.01) $\mathrm{ms} ; F(1,14)=4.73, p<0.05)$. The effect of Visual Field was clearly not significant $(F<1)$, but the category by visual field interaction approached significance $F(1,14)=3.68, p=0.076$. The impression of an almost significant interaction was supported by paired samples $t$-tests (2-tailed) used to investigate the interaction. There was a significant category effect for the RVF $(t(14)=2.97, p<0.05)$, but not for the LVF $(t(14)=0.41, p=0.69)$. There was no significant difference across visual fields for cross-category responses $(t(14)=0.21, p=0.84)$ or within-category responses $(t(14)=$ $1.21, p=0.26$.

\section{Discussion}

The characteristic pattern of lateralisation of $\mathrm{CP}$ was found 
again in this experiment, although the crucial category by visual field interaction did not quite reach significance. There was clearly no category effect in the LVF, but between-category was about $15 \mathrm{~ms}$ faster than within-category in the RVF.

In addition, there was no suggestion that the number of distractors affected RTs, indicative of pop-out or efficient search, nor any suggestion that the number of distractors affected the pattern of lateralisation. Thus with the caveat that the category by visual field interaction was not quite significant, these data provide further support for the LH bias in colour $\mathrm{CP}$.

\section{EXPERIMENT 2: HEMISPHERIC ASYMMETRIES IN COLOUR CP IN A PRESENT-AbSENT TARgET DETECTION TASK}

In Experiment 1, lateralisation of colour $\mathrm{CP}$ was investigated using a visual search task where the number of distractors varied. The findings showed a trend towards the $\mathrm{LH}$ bias in colour CP found in previous research requiring a left-right target location decision as in Gilbert et al. 's search task, and Drivonikou et al. 's [15]. target detection task. The results showed that the pattern of lateralisation was independent of the number of distractors, confirming that, detecting a target colour amongst differently coloured distractors is a 'pop-out' task, and confirming that the LH bias is invariant across basic changes in the nature of the task.

As a further extension of the range of tasks used to test the robustness of the $\mathrm{LH}$ bias, Experiment 2 used a target detection task, as in Gilbert et al., [14], but with the modification that the decision required did not involve an explicit spatial component, [14], [15]. Instead, a target was only present on half the trials, and the decision required was 'target-present' versus 'target-absent'.

\section{A. Method}

\section{1) Participants}

Participants were twenty-four native English-speaking undergraduates recruited from the student population of the University of Surrey. There were 5 males with a mean age of 19.00 years $(\mathrm{SD}=0.71)$, and 19 females, with a mean age of 18.90 years $(\mathrm{SD}=1.59)$, with an age range from 18 to 24 years old. Based on self-report, all were right-handed and had normal colour vision, as indicated by the City University Test [26]; all participated for course credit, and none of them were aware of the predictions of the experiment at the time of testing.

\section{2) Stimuli, design and apparatus}

Four colours were selected for this experiment as shown in Fig. 4. Two stimuli were blue and two were green. They varied only in Munsell hue: 10G, 5BG, 10BG and 5B, with value and chroma kept constant (6/7). The separation between stimuli was 5 hue steps $(\Delta E \sim 15)$. Their CIELUV coordinates $\left(u^{*} v^{*}\right)$ were: $-43.64,12.12 ;-45.65,-2.73 ;-44.83$, -18.68; 39.62, 32.97. A Cambridge Research Instruments Colour Cal was used to measure CIE co-ordinates. The colour stimuli were displayed on calibrated 17 inch CRT Sony Trinitron monitor.

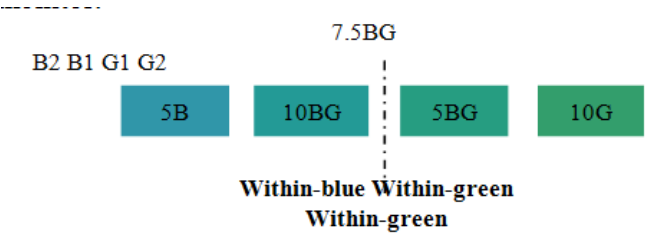

Fig. 4. Illustration of the Munsell codes of the four stimuli used. B2 and B1 are blue and G1 and G2 are green. Adjacent colours are 5 hue steps apart. The line between B1 and G1 indicates the English blue-green boundary.

\section{3) Procedure}

The target was a circle of $3 \mathrm{~cm}$ diameter (visual angle $=$ $\left.3.22^{\circ}\right)$ that appeared on a differently coloured background $(40 \times 30 \mathrm{~cm})$ at one of twelve locations around a central fixation point, with half of to the right of fixation and half to the left of fixation (see Fig. 5). There were three target-background pairs: two within-category (blue1-blue2 and green1-green2) and one between category (blue1-green1). Within each pair, half the time, one was the target and the other the background, and on the other half of the trials, the relationship was reversed. There were 192 trials in total, half target present and half target absent. The 96 target present trials consisted of 24 trials for each of the combinations of visual field (LVF RVF) and category (within- between-). Trial order was randomised subject to the above constraints.

A

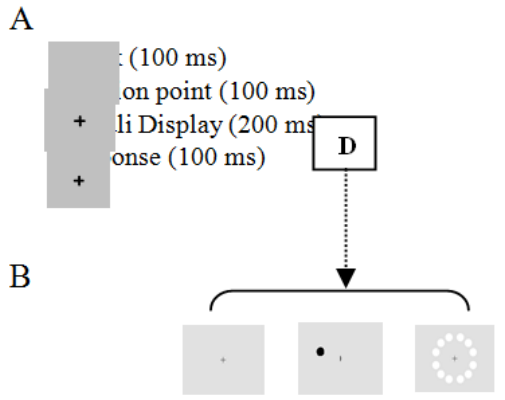

Target absent Target present Target locations

Fig. 5. a) Example display of the target detection task. b) First left indicates the display when the target absent, display in the middle indicates the target present and first right showed 12 white circles indicates the place that the target could appear.

The experiment was conducted in a dark room. Participants viewed the display at a distance of $60 \mathrm{~cm}$, and their head was restrained by using a chin rest. They were instructed that on approximately half the trials there would be a coloured target on a coloured background. On the remaining trials, there would be no target. When there was a target, it could occur, at random, in any one of 12 locations arranged on a notional circle around the fixation cross. Their task was to decide on each trial whether the target was present or absent and to respond by pressing the left or right mouse button, as appropriate. For half the subjects, the left button indicated target present and for the other half, the right button indicated target present.

\section{B. Results}

As the main interest is in lateralised CP, I only report the analysis of target present trials; the within-between category and the visual field variables only apply to target present trials. 
Overall, there were only $2.3 \%$ errors; too small a rate to be usefully analysed. For each participant, the median RT for correct trials, for each combination of visual field and category was calculated. Fig. 6 shows the mean RT across participants for each combination of category (within/cross) and visual field (left/right). A repeated-measures two-way ANOVA showed that between-category (mean $(\mathrm{SD})=558$ (65.13) ms) was about $21 \mathrm{~ms}$ faster than within-category $($ mean $(\mathrm{SD})=579(73.71) \mathrm{ms} ; F(1.23)=27.83, p<0.001)$.

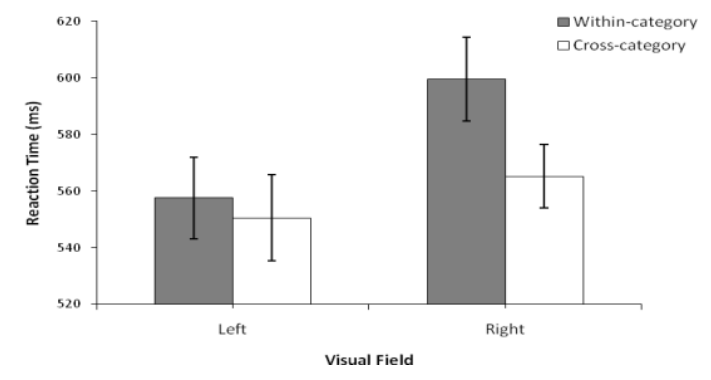

Fig. 6. Median response times ( $\mathrm{ms}+/-1 \mathrm{se}$ ) for correct trials for detecting of chromatic target on colour background. The data were averaged over each combination of category (within/across) and visual fields (LVF/RVF).

RVF RTs (mean (SD) $=582(65.58) \mathrm{ms})$ were about $28 \mathrm{~ms}$ slower than LVF RTs (mean $(\mathrm{SD})=554(72.0) ; F(1,23)=$ 27.83, $p<0.001)$. And, crucially, there was also an interaction between Category and Visual Field $(F(1,23)=$ $4.72, p<0.05)$. From Fig. 6, it appears that the interaction probably reflects a category effect in the RVF while there is probably no LVF category effect. Paired sample $t$-tests supported this impression: RVF, $t(25)=3.85, p<0.001 ; \mathrm{LVF}$, $t(25)=0.82, p=0.42$. Additionally, the main reason for the difference in the $\mathrm{CP}$ in the two visual fields seems to be due to the particularly high LVF RTs: there was no significant visual field effect for between-category, $(t(25)=1.63, p=$ $0.12)$ whereas there was for within-category $(t(25)=5.62, p$ $<0.001)$.

\section{Discussion}

The resuls mirrored the finding of previous experiments where discrimination of pairs of colour from different lexical categories (blue and green) was faster than pairs from the same lexical category (different shades of blue), particularly when the two colours were presented to the RVF (LH).

The aim of the experiment was to replicate the original finding of LH lateralised colour CP using a task that varied in design and instructions to the tasks used in previous research. Participants were asked to decide if there was a target on a different coloured background, without taking into account the target location, when there was a target on just half of the trials. Response times showed the size of the category effect was significantly larger for the RVF than for the LVF suggesting that LH bias for CP still occurs when no explicit spatial decision is required. This result provides converging evidence that colour $\mathrm{CP}$ is lateralised to the RVF (LH).

\section{General Discussion OF THE CHAPTER}

The overall aim of the experiments presented in this paper was to assess whether previous findings of $\mathrm{LH}$ lateralised colour $\mathrm{CP}$ are independent of reading direction, the number of distractors and the nature of the target-decision. In brief, the 'robustness' of the effect. The previous studies that found LH colour CP, [14], [15], [18], [20], [22], [24] have all tested participants who read left-to-right scripts (or top to bottom scripts), but participants who read from right-to-left had not been tested. Reading direction affects the pattern of lateralisation on a range of perceptual tasks [27]-[32], so it was plausible that reading direction could affect the lateralisation of colour CP. This hypothesis was tested by Al-rasheed et al. [17] by testing the lateralisation of blue-green colour $\mathrm{CP}$ in Arabic participants. The lateralisation of colour CP was investigated in A-rasheed for English and Arabic speakers - two groups of participants who differ in their reading direction. A visual search task with an RT measure and a target detection task with an initiation time eye-movement measure have been used. It was found that both groups had colour CP that was stronger in the LH than the RH. This confirms the robust nature of the lateralisation of colour $\mathrm{CP}$ to the $\mathrm{LH}$.

Lateralised colour CP was also tested in Experiments 1 and 2 in the current study using two types of task that were different to the tasks used in previous research, [14]-[17], [22]-[24]. This allowed a further check on the robustness of the LH category bias. There was a significant LH bias in colour CP on a present/absent target detection task. However, on a visual search task where stimuli were shown in a grid array with a varying number of distractors, there was only a trend for a LH bias. This may suggest that some tasks are better than others at eliciting LH colour CP bias. However, the overall impression from this set of experiments is that $\mathrm{LH}$ colour CP is a robust effect that generalises across participants with different reading directions and across different types of visual search and target detection tasks.

In summary, the present results of this paper revealed LH lateralisation of colour CP. The LH bias in colour CP appears not to be affected by reading direction as in Al-rasheed [18], and the effect is found (or there is a trend for the bias) on a range of different visual search and target detection tasks.

\section{REFERENCS}

[1] S. Harnad, Categorical Perception: The Groundwork of Cognition, New York: Cambridge University Press, 1987.

[2] K. Uchikawa and H. Shonida. (1996). Influence of Basic Colour Categories on Colour Memory Discrimination. Colour Research and Application. [Online]. 21. pp. 430-439. Available: http://dx.doi.org/10.1002/(SICI)1520-6378(199612)21:6<430::AID-C OL5>3.0.CO;2-X

[3] D. Roberson, J. Davidoff, and N. Braisby. (1999). Similarity and Categorisation: Neuropsychological Evidence for a Dissociation in Explicit Tasks. Cognitio. [Online]. 71. pp. 1-42. Available: http://dx.doi.org/10.1016/S0010-0277(99)00013-X

[4] D. Roberson and J. Davidoff. (2000). The Categorical Perception of Colours and Facial Expressions: The Effect of Verbal Interference. Memory and Cognition. [Online]. 28. pp. 977-986. Available: http://dx.doi.org/10.3758/BF03209345

[5] D. Roberson, I. R. L. Davies, and J. Davidoff. (2000). Colour Categories Are Not Universal: Replications and New Evidence from a Stone-Age Culture. Journal of Experimental Psychology: General, [Online]. 129, pp. 369-398. Available: http://dx.doi.org/10.1037/0096-3445.129.3.369

[6] M. Pilling, A. Wiggett, E. Özgen, and I. R. L. Davies. (2003). Is Colour "Categorical Perception" Really Perceptual?. Memory and Cognition. [Online]. $31 . \quad$ pp. 538-551. Available: http://dx.doi.org/10.3758/BF03207082 
[7] M. H. Bornstein and N. Korda. (1984). Discrimination and Matching within and between Hues Measured by Reaction Times: Some Implications for Categorical Perception and Levels of Information Processing. Psychological Research. [Online]. 46. pp. 207-222. Available: http://dx.doi.org/10.1007/BF00308884

[8] R. M. Boynton, L. Fargo, C. X. Olson, and H. S. Smallman. (1989). Category Effect in Colour Memory. Colour Research and Application. [Online]. $14 . \quad$ pp. 229-234. Available: http://dx.doi.org/10.1002/col.5080140505

[9] G. Laws, I. Davies, and C. Andrews. (1995). Linguistic Structure and Non-Linguistic Cognition: English and Russian Blues Compared. Language and Cognitive Processes. [Online]. 10. pp. 59-94. Available: http://dx.doi.org/10.1080/01690969508407088

[10] A. Franklin, M. Pilling, and I. R. L. Davies. (2005). The Nature Colour Categorisation: Evidence from Eye-Movements on a Target Detection Task. Journal of Experimental Child Psychology. [Online]. 91. pp. 227-248. Available: http://dx.doi.org/10.1016/j.jecp.2005.03.003

[11] C. A. Daoutis, A. Franklin, A. Riddet, A. Clifford, and I. R. L. Davies. (2006). Categorical Effects in Children's Colour Search: A Cross-Linguistic Comparison. British Journal of Developmental Psychology. [Online]. 24. pp. 373-400. Available: http://dx.doi.org/10.1348/026151005X51266

[12] C. A. Daoutis, M. Pilling, and I. R. L. Davies. (2006). Categorical Effects in Visual Search of Colour. Visual Cognition. [Online]. 14. pp. 229-234. Available: http://dx.doi.org/10.1080/13506280500158670

[13] A. S. A. rasheed. "Categorical perception of colour: the background of perception," in Proc. Workshop and International Conference on Measurement in Education (WICME), Jakarta, vol. 1. pp. 209-229, 2012.

[14] A. L. Gilbert, T. Regier, P. Kay, and R. B. Ivry, "Whorfian hypothesis supported in the right visual field but not the left," Proceeding of the National Academy of Sciences, 2006, vol. 103, 489-494.

[15] G. V. Drivonikou, T. Kay, R. B. Regier, A. L. Ivry, A. Franklin, and I. R. L. Davies. (2007). Further Evidence That Whorfian Effects Are Stronger in the Right Visual Field than the Left. Proceeding of the National Academy of Sciences. [Online]. 104. pp. 1097-1102. Available: Available: http://dx.doi.org/10.1073/pnas.0610132104

[16] D. Roberson, H. Park, and R. J. Hanley. (2008). Categorical Perception of Colour in the Left and Right Visual Field Is Verbally Mediated: Evidence from Korean. Cognition. [Online]. 107. pp. 752-762. Available: http://dx.doi.org/10.1016/j.cognition.2007.09.001

[17] A. S. A. rasheed, A. Franklin, G. V. Drivonikou, and I. R. L. Davies. (2014). Left Hemisphere Lateralization of Categorical Colour Perception among Roman and Arabic Script Readers. Journal of Psychology. [Online]. 3. pp. 1-16. Available: http://www.scirp.org/journal/psych

[18] G. V. Drivonikou, I. R. L. Davies, A. Franklin, and C. Taylor, "Lateralisation of colour categorical perception: a cross-cultural study," Perception, vol. 36, 2007.

[19] A. Franklin, G. V. Drivonikou, L. Bevis, I. R. L. Davies, P. Kay, and T. Regier. (2008). Categorical Perception of Colour IsLateralised to the Right Hemisphere in Infants, But to the Left Hemisphere in Adults. Proceedings of the National Academy of Sciences. [Online]. 105. pp. 3221-3225. Available: http://dx.doi.org/10.1073/pnas.0712286105

[20] W. T. Siok, P. Kay, W. S. Y. Wang, A. H. D. Chan, L. Chen, K. Luke, and L. H. Tan, "Language Regions of Brain Are Operative in Colour Perception," Journal of Experimental Psychology: Human Perception and Performance, vol. 106, pp. 8140-8145, 2009.

[21] Q. Liu, H. Li, J. L. Campos, Q. Wang, Y. Zhang, J. Qiu, Q. Zhang, and H. Sun. (2009). The N2pc Component in ERP and Lateralization Effect of Language on Colour Perception. Neuroscience Letters. [Online]. $454 . \quad$ pp. 58-61. Available: http://dx.doi.org/10.1016/j.neulet.2009.02.045

[22] A. Treisman, and G. Gelade, "A feature integration theory of attention," Cognitive Psychology, vol. 12, pp. 97-136, 1980.

[23] J. Wolfe, "Guided search 2.0: a revised model of visual search," Psychonomic Bulletin and Review, vol. 1, pp. 202-238, 1994.

[24] A. Franklin, G. V. Drivonikou, A. Clifford, P. Kay, T. Regier, and I. R. L. Davies. (2008). Laterlization of Categorical Perception of Colour Changes with Colour Term Acquisition. Proceedings of the National Academy of Sciences. [Online]. 47. pp. 18221-18225. Available: http://dx.doi.org/10.1073/pnas.0809952105

[25] M. H. Bornstein and M. D. Monroe, "Chromatic information processing: rate depends on stimulus location in the category and psychological complexity," Psychological Research, vol. 42, pp. 213-225, 1980

[26] R. Fletcher, the City University Colour Vision Testm, Windsor, Berks: Keeler Ltd, 1980.

[27] Z. Eviatar. (1995). Reading Direction and Attention: Effects on Lateralized Ignoring. Brian and Cognition. [Online]. 29. pp. 137-150. Available: http://dx.doi.org/10.1006/brcg.1995.1273

[28] Z. Eviatar, "Language experience and right hemisphere task: the effects of scanning habits and multilingualism," Brian and Cognition, vol. 58, pp. 157-173, 1997 .

[29] M. Farid and J. Grainger. (1996). How Initial Fixation Position Influences Visual Word Recognition: A Comparison of French and Arabic. Brian and Language. [Online]. 53. pp. 351-368. Available: http://dx.doi.org/10.1006/brln.1996.0053

[30] J. F. Prunet, R. Beland, and A. Idrissi, "The mental representation of Semitic word," Linguistic Inquiry, vol. 31, pp. 609-648. 2000.

[31] I. Berent. (2002). Identity Avoidance in the Hebrew Lexicon Implications for Symbolic Accounts of Word Formation. Brian and Language. [Online]. 81. pp. 326-341. Available: http://dx.doi.org/10.1006/brln.2001.2528

[32] N. D. Schwalm, Z. Eviatar, Y. Golan, and Y. Blumenfeld. (2003). The Effect of Reading Direction Habit on Numerical Processing. Proceeding of the Human Factors and Ergonomics Society. [Online] pp. 1649-1653. Available: http://dx.doi.org/10.1177/154193120304701312

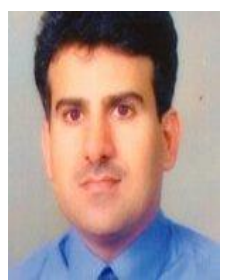

Abdulrahman Al-Rasheed completed his doctorate under the supervision of professor Ian Davies and Anna Franklin at the University of Surrey after taking up his first academic position as a lecturer at KSU 2003. He is currently an assistant professor at the Department of Psychology, King Saud University.

Abdul's research aim is to investigate the contribution of language to categorization, also to explore categorization across variations languages using behavioral approaches such as eye-tracking and measures of accuracy and reaction time, as well as an electrophysiological method called the Event-Related Potential (ERP) technique currently he involved with several research studies. These include: 1) Cross-cultural differences in colour perception and cognition. 2) Effect of language on cognitive processes. 3) Factors underlying colour preference. 4) The effect of reading directions in categorization and cognition 